\title{
Blumea Balsamifera (Sambong) Tea as a Therapeutic Drink for Calcium Oxalate Stones
}

\author{
Charlimagne M. Montealegre ${ }^{1, a}$, Rizalinda L. De Leon ${ }^{1}$ \\ ${ }^{1}$ Department of Chemical Engineering, College of Engineering, University of the Philippines, 1101 Diliman Quezon City
}

\begin{abstract}
Calcium oxalate stones are among the most prevalent type of kidney stones. Methods of treatment range from surgical removal, shockwave lithotripsy, medical and natural treatment. In the Philippines, consuming tea preparation of Blumea balsamifera is believed to aid in the treatment of kidney stones. The effect of Blumea balsamifera tea in the nucleation of calcium oxalate crystals was studied at $0,0.5$ and $1.0 \mathrm{mg}$ tea preparation per $\mathrm{mL}$ of the crystallization solution by UV-Vis spectrophotomery. Induction time decreased with increasing concentration of Blumea balsamifera tea. Further analysis showed that this decrease in induction time is due to a decrease in surface free energy from $20.95 \mathrm{~mJ} / \mathrm{m}^{2}$ to $19.48 \mathrm{~mJ} / \mathrm{m}^{2}$ and $17.22 \mathrm{~mJ} / \mathrm{m}^{2}$ at 0.5 and $1.0 \mathrm{mg} / \mathrm{mL}$, respectively. This decrease in induction time correspond to an increased nucleation rate thus, a large number of smaller crystals are formed. This explains the therapeutic benefits of consuming Blumea balsamifera tea as stone formers would form smaller stones that are easier to eliminate by urination.
\end{abstract}

\section{Introduction}

Plants serve a variety of applications including food and as naturopathic agents. Its phytochemicals contain medicinal properties which not only improves but also augments health. There exists a large market for naturopathic plants consumed as food supplement capsules, in its raw form, or preparations as juices and tea.

The benefits of consuming herbal teas and decoction of leaves and other plant parts are widely recognized for the management and treatment of various ailments including kidney stones. This method of treatment does not only increase urinary volume but also provides phytochemicals which serve various purposes. The study by Rodgers et al showed that though urine volume (together with 12 other parameters) significantly influences stone formation, they key difference between stone formers and non-stone formers is the speciation of calcium ions in the urine [1]. Speciation is influenced primarily by an individual's metabolism and also by the presence of crystal growth modifiers acting as promoters and inhibitors of stone formation.

The crystal growth modifying property of various compounds are available in the literature. Some of these are inorganic salts such as citrate, magnesium and pyrophosphate [2]. These compounds were able to inhibit the crystallization of calcium oxalate by different means. Citrate forms a complex with calcium ions while pyrophosphate, to a certain extent, is incorporated into the forming crystal [3]. Biomolecules such as Tammhorsfall protein, Uropontin, glycosaminoglycans, Bovine serum albumin, chondrotoin sulfate etc. also affects the crystallization process by modifying the habit of the crystal. Bovine serum albumin binds the $\left\{\begin{array}{lll}1 & 2 & -1\end{array}\right\}$ and $\{0$ 21 face of the growing crystal changing the shape of the calcium oxalate crystal from hexagonal to diamondshaped [4]. On the other hand, chondrotoin sulfate binds the $\left(\begin{array}{lll}0 & 1 & 0\end{array}\right)$ face resulting to elongated hexagonal crystals [4].

Compounds from plant such as quercetin, hyperoside [5] and crude plant extracts were also reported to inhibit the formation of calcium oxalate crystals. An in vivo study by Zhu et al. showed a significant decrease in crystal deposits for quercetin and hyperoside treated rats [5]. The extract of Khella or Ammi visnagia is used as an herbal remedy in Egypt, Middle East around Mediterranean and in South Africa. Extract from the seeds of Khella were shown to decrease the surface free energy and shift the morphology of calcium oxalate crystals from the monohydrate to calcium oxalate dihydrate [6]. A similar shift in morphology was shown by the extract of Bergenia ciliata, and was shown to be more effective in inhibiting nucleation and aggregation of COM crystals when compared with cystone [7]. Alok et al listed a total of 82 plants and their parts traditionally regarded to be herbal drugs used in the treatment of kidney stone, gall stone and urinary stone [8].

In the Philippines, Blumea balsamifera is recommended by the National Kidney Transplant Institute as an herbal remedy for kidney stones. Early studies found a statistically significant dissolution between the control and stones treated with urine from individuals taking $B$. balsamifera tablet [9] but the effect

\footnotetext{
a Corresponding author: charlimagne.montealegre@coe.upd.edu.ph
} 
on the crystallization of calcium oxalate stones was unknown.

Crystallization is a complex process involving nucleation, crystal growth, and aggregation. Dissolution complicates the process further since these processes may occur simultaneously. In classical nucleation theory, the rate of nucleation is modeled in Arrhenius form

$$
J=A \exp \left(-\frac{\Delta G}{k T}\right)
$$

A thermodynamic assessment of the Free energy of formation of a stable nuclei $(\Delta G)$ shows that the free energy of nucleation is a function of surface free energy $(\gamma)$ and supersaturation $(s)$ shown as:

$$
\Delta G=\frac{16}{3} \pi \gamma^{3} \frac{v^{2}}{k^{2} T^{2}(\ln s)^{2}}
$$

The rate of nucleation is difficult to measure due to the variations on the size of a nuclei which may vary from ten to several thousand molecules [10]. A more measurable parameter is induction time. Induction time is inversely proportional to the rate of nucleation. Thus converting to an equality using a proportionality constant k',

$$
t_{\text {ind }}=\frac{k^{\prime}}{J}=\frac{k^{\prime}}{A} \exp \left(\frac{16}{3} \pi \gamma^{3} \frac{v^{2}}{k^{3} T^{3}(\ln s)^{2}}\right)
$$

This may be linearized by taking the natural logarithm of the equation.

$$
\ln t_{\text {ind }}=\ln k^{\prime \prime}+\frac{16}{3} \pi \gamma^{3} \frac{v^{2}}{k^{3} T^{3}(\ln s)^{2}}
$$

Equation 4 shows that a plot of the natural logarithm of the induction time and the reciprocal of the square of the natural logarithm of supersaturation will give a straight line and surface free energy may be calculate from the slope of this line.

This study aims to provide evidence on the effect of Blumea balsamifera tea on the crystallization of calcium oxalate through the induction time.

\section{Materials and Methodology}

Reactive crystallization is performed by mixing equal concentrations of calcium and oxalate solutions. Calcium solution is prepared from analytical grade calcium chloride dihydrate and Oxalate solution, from Sodium oxalate. These solutions are diluted using deionized water.

Blumea balsamifera decoction was prepared by boiling Golden Spoon ${ }^{\mathrm{TM}}$ all natural sambong tea in water for 5 minutes. The decoction was filtered through cellulose filter paper, cooled to room temperature and diluted to proper volume.

Induction time is the time difference between the mixing of a supersaturated solution and the detection of crystals in the solution. This parameter is inversely proportional to nucleation rate. Induction time is measured by monitoring the absorbance of the supersaturated solution using Perkin-Elmer Lambda 850 at $560 \mathrm{~nm}$ as optimized by a previous study [6]. An algorithm is used to systematically determine the induction time. An approximation of induction time is made by visual inspection of the spectra. The first derivative plot reflects the rate of change of the absorbance. When the changes in absorbance are gradual the first derivative plot is constant and an abrupt change is determined by a change in the slope of the first derivative plot. As the slope of the first derivative plot changes, the second derivative plot would also exhibit a sudden change or a jump discontinuity. The maximum of the second derivative plot is determined numerically to determine the time corresponding to the sudden change in the first derivative plot and consequently the induction time. Due to noise in the data, Savitzky-Golay smoothening is used.

To determine the effect of the decoction on the rate of nucleation, the degree of supersaturation is varied from 10 to 20 with increments of 2.5 The degree of supersaturation is based on the solubility of Calcium oxalate monohydrate at $25^{\circ} \mathrm{C}$ calculated using equation 5 [11]:

$$
s=\sqrt{\frac{\left[\mathrm{Ca}^{2+}\right]\left[\mathrm{C}_{2} \mathrm{O}_{4}^{2-}\right]}{K_{s p, 25^{\circ} \mathrm{C}}}}
$$

The level of decoction was changed from a blank, 0.5 and $1.0 \mathrm{mg}$ Blumea balsamifera tea preparation per $\mathrm{mL}$ of the crystallization solution.

\section{Results and Discussion}

The effect of Blumea balsamifera tea on the crystallization of calcium oxalate crystals is studied by determining the effect on induction time. Figure 1 shows absorbance-time plot at different levels of extract.

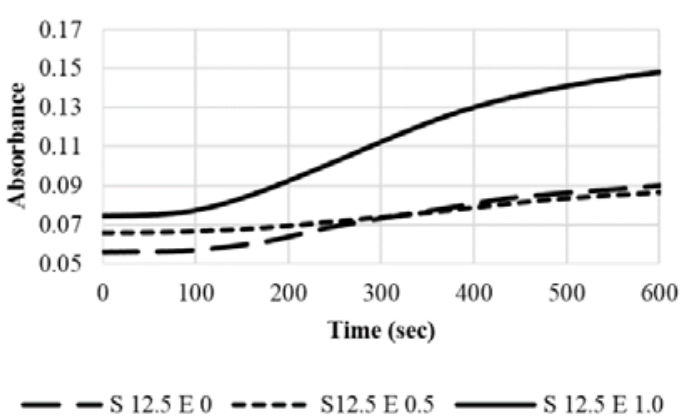

Figure 1. Absorbance versus time plot for a supersaturation of 12.5 and varying decoction concentrations shows an increase in induction time with increased concentration

The induction time is defined to be the point in time where there is a sudden increase in the absorbance from the initial absorbance measurements. This sudden increase is due to the formation of a stable absorbing specie, i.e. crystal formation. The absorbance increases continuously as the crystals grow. In some systems the absorbance decrease due to flocculation of the crystals 
formed. Figure 1, also shows that with an increase in extract concentration, the induction time decreases, i.e. stable crystals are formed faster and nucleation rate increases.

Induction time is plotted with respect to the reciprocal of the square of the natural logarithm of the supersaturation according to equation 4 . These plots are summarized in Figure 2A for $0 \mathrm{mg} / \mathrm{mL}, 2 \mathrm{~B} 0.5 \mathrm{mg} / \mathrm{mL}$ and Figure 2C for $1.0 \mathrm{mg} / \mathrm{mL}$ decoction. The surface free energy is computed from the slope of the plots and are summarized in the Table 1.

Table 1. Summary of slope, Pearson correlation factor and calculated Surface free energy.

\begin{tabular}{|c|c|c|c|}
\hline Parameter & Slope & RSQ & $\gamma\left(\mathrm{mJ} / \mathrm{m}^{2}\right)$ \\
\hline Blank & 26.901 & 0.644 & 20.95 \\
\hline $0.5 \mathrm{mg} / \mathrm{mL}$ & 21.618 & 0.642 & 19.48 \\
\hline $1.0 \mathrm{mg} / \mathrm{mL}$ & 14.929 & 0.147 & 17.22 \\
\hline
\end{tabular}

The surface free energy of the system decreases upon the addition of the extract, consistent with previously published studies [6]. There was a 7.03\% decrease in the surface free energy at an extract concentration of 0.5 $\mathrm{mg} / \mathrm{mL}$ and a $17.82 \%$ decrease observed with the higher extract concentration of $1.0 \mathrm{mg} / \mathrm{mL}$. The calculated surface free energy from the induction time studies was compared with a theoretical surface free energy using the proposed correlation of Mersmann [10].

$$
\gamma=0.414 k T\left(\frac{\rho_{C} N}{M}\right)^{2 / 3} \ln \frac{C_{S}}{C_{L}}
$$

The proposed correlation of Mersmann enables the theoretical determination of surface free energy from the Boltzmann constant $k$, Avogadro's number $N$, molar mass of the crystal $M$, crystal density $\rho_{C}$, solute concentration in the solid phase $C_{S}$ and solute concentration in the liquid phase $C_{L}$. However, the experimentally determined surface free energy differ from the Mersmann correlation predicted value by $71.82 \%$. The correlation of Mersmann is under the simplification that surface free energy is only a function of relative supersaturation and material properties. In reality, the surface free energy varies across each crystal face, crystal edge, and within the surface as considered by more recent crystal growth theories such as the Burton-Cabrera-Frank theory, herring etc.

The critical radius of nuclei formation and change in critical Gibb's free energy were calculated and shown in Figures 3 and 4. Both the critical Gibb's free energy and critical nuclei radius decreased with the addition of the extract. At $0.5 \mathrm{mg} / \mathrm{mL}$ of extract, critical radius decreased by $7.03 \%$ while at $1.0 \mathrm{mg} / \mathrm{mL}$ the percentage of reduction was higher at $17.82 \%$, across all degrees of supersaturation. Thus, a smaller nuclei size is required to form a stable crystal for growth.

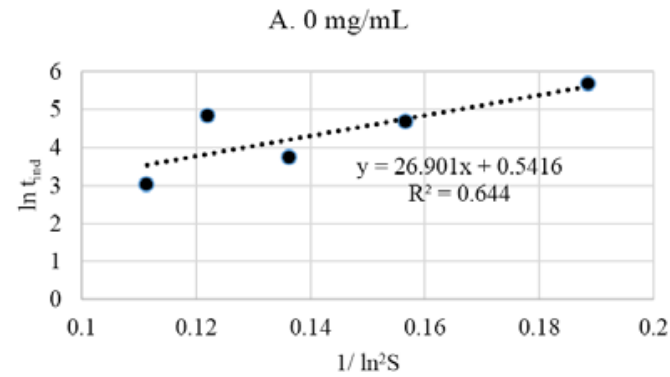

B. $0.5 \mathrm{mg} / \mathrm{mL}$

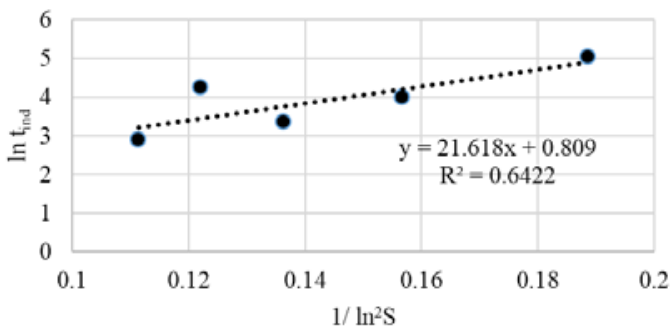

C. $1.0 \mathrm{mg} / \mathrm{mL}$

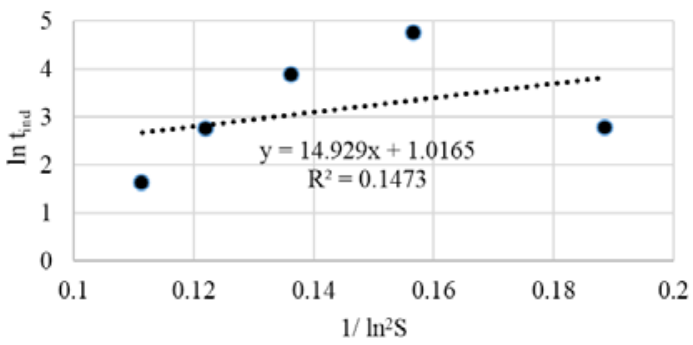

Figure 2. Variation of the induction time with respect to supersaturation at A 0, B 0.5 and C $1.0 \mathrm{mg} / \mathrm{mL}$ Blumea balsamifera tea preparation

The critical Gibbs' free energy decreased by $19.64 \%$ and $44.50 \%$ at 0.5 and $1.0 \mathrm{mg} / \mathrm{mL}$ of extract, respectively. The decrease in the critical Gibbs' free energy for the nucleation process shows that in the presence of the extract the formation of stable nuclei is easier. Nucleation is therefore promoted by the addition of the extract.

Addition of the extract decreased the induction time, free surface energy, decreased the critical Gibb's free energy and critical radius of a stable nuclei. All of these results support the observation that nucleation is promoted forming a large number of crystals that are smaller in size.

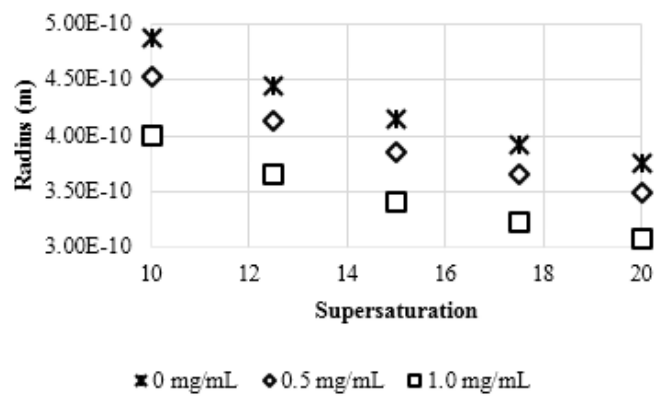

Figure 3. The critical radius of the nuclei decreases with increasing degree of supersaturation and with an increasing decoction concentration 


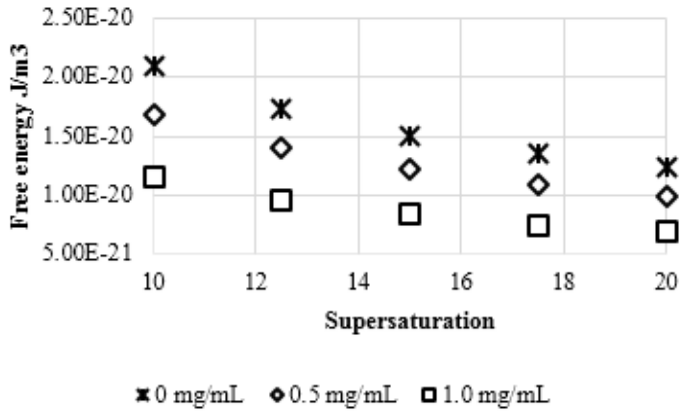

Figure 4. Critical Gibbs' free energy decreased as concentration is increased

\section{Conclusion}

A decoction of Blumea balsamifera tea preparation was shown to reduce the induction time thus increasing the nucleation rate. The surface free energy decreases with the addition of the extracts from $20.95 \mathrm{~mJ} / \mathrm{m}^{2}$ without the extract to $19.49 \mathrm{~mJ} / \mathrm{m}^{2}$ at $0.5 \mathrm{mg} / \mathrm{mL}$ and $17.21 \mathrm{~mJ} / \mathrm{m}^{2}$ at $1.0 \mathrm{mg} / \mathrm{mL}$ of Blumea balsamifera tea. This corresponds to a $7.03 \%$ and $17.82 \%$ reduction in surface free energy at 0.5 and $1.0 \mathrm{mg} / \mathrm{mL}$, respectively. The calculated critical nuclei radius also decreased by $7.03 \%$ at 0.5 $\mathrm{mg} / \mathrm{mL}$ and $17.82 \%$ at $1.0 \mathrm{mg} / \mathrm{mL}$. Critical Gibbs free energy also decreased with increasing extract concentration showing that the nucleation process is favored. An increased nucleation rate implies an increased number of crystals that are smaller in size. This effect of the extract explains the therapeutic effect of consuming Blumea balsamifera tea as a therapeutic drink as stone formers would form smaller stones that are easier to eliminate by urination.

\section{Compliance with Ethical Standards}

The authors declare that they have no conflict of interest. This article does not contain any studies with human participants or animals performed by any of the authors.

\section{References}

[1] A.L. Rodgers, S. Allie-Hamdulay, G.E. Jackson, I. Durbach, J. Cryst. Growth 382 (2013) 67-74.

[2] M. Gupta, S. Bhayana, S.K. Sikka, Int. J. Res. Pharm. Chem. 1 (2011) 793-798.

[3] J.A. Muñoz, M. López-Mesas, M. Valiente, Urology 80 (2012) 1163.e13-8.

[4] S. Farmanesh, S. Ramamoorthy, J. Chung, J.R. Asplin, P. Karande, J.D. Rimer, J. Am. Chem. Soc. 136 (2014) 367-76.

[5] W. Zhu, Y.-F. Xu, Y. Feng, B. Peng, J.-P. Che, M. Liu, J.-H. Zheng, Urolithiasis (2014).

[6] E. a. Abdel-Aal, S. Daosukho, H. El-Shall, J. Cryst. Growth 311 (2009) 2673-2681.

[7] S. Saha, R.J. Verma, Arab J. Urol. 11 (2013) 187192.

[8] S. Alok, S.K. Jain, A. Verma, M. Kumar, M. Sabharwal, Asian Pacific J. Trop. Dis. 3 (2013) 496504.

[9] F. Rico, Philipp. J. Urol. 2 (1992) 9-13.

[10] J.W. Mullin, Crystallization, Elsevier, 2001.

[11] M. Öner, A. Khan, S.R. Khan, in:, Miner. Scales Depos., Elsevier, 2015, pp. 393-416. 